\title{
The Long Noncoding RNA RPS1OP2-AS1 Is Implicated in Autism Spectrum Disorder Risk and Modulates Gene Expression in Human Neuronal Progenitor Cells
}

\author{
Stephanie M. Bilinovich ${ }^{1}$, Kristy Lewis ${ }^{1}$, Nicole Grepo ${ }^{2}$ and Daniel B. Campbell ${ }^{\text {* }}$ \\ ${ }^{1}$ Department of Pediatrics and Human Development, Michigan State University, Grand Rapids, MI, United States, \\ ${ }^{2}$ Center for Gene Therapy, City of Hope, Duarte, CA, United States
}

OPEN ACCESS

Edited by:

Alika Maunakea,

University of Hawaii,

United States

Reviewed by:

Nasun Hah,

Salk Institute for Biological Studies,

United States

Shicheng Guo,

Marshfield Clinic Research Institute,

United States

* Correspondence:

Daniel B. Campbell

Daniel.Campbel/@hc.msu.edu

Specialty section:

This article was submitted to Epigenomics and Epigenetics,

a section of the journal

Frontiers in Genetics

Received: 17 June 2019 Accepted: 11 September 2019

Published: 15 October 2019

Citation:

Bilinovich SM, Lewis K, Grepo N and Campbell DB (2019) The Long

Noncoding RNA RPS1OP2-AS1

Is Implicated in Autism Spectrum

Disorder Risk and Modulates Gene

Expression in Human Neuronal

Progenitor Cells.

Front. Genet. 10:970.

doi: 10.3389/fgene.2019.00970
Most of the genetic risk for autism spectrum disorder (ASD) is inherited as common genetic variants, although some rare mutations have been identified in individuals with ASD. Common genetic variants are most parsimoniously identified by genome wide association studies. Genome wide association studies have identified several genetic loci with genome wide association with ASD. However, genome wide association studies only identify regions of the genome associated with phenotypic traits. Identification of the functional elements requires additional experimental evidence. Here, we demonstrate that a genome wide association study locus for ASD on chromosome 20p12.1, rs4141463, implicates a noncoding RNA as a functional element. Although rs4141463 lies within an intron of the protein-coding MACROD2 (MACRO domain containing 2) gene, expression of MACROD2 is neither altered in postmortem temporal cortex of individuals with ASD nor correlated with rs4141463 genotype. Our bioinformatics approaches revealed a noncoding RNA transcript near the autism susceptibility signal, RPS10P2-AS1 (ribosomal protein S10 pseudogene 2 anti-sense 1). In a panel of 15 human tissues, RPS10P2-AS1 was expressed at higher levels than the protein-coding MACROD2 in both fetal temporal cortex and adult peripheral blood. In postmortem temporal cortex, expression of RPS10P2-AS1 was increased 7-fold in individuals with ASD $(P=0.02)$ and increased 8 -fold in individuals with the ASD-associated rs4141463 genotype $(P=0.01)$. Further, RPS10P2-AS1 expression was increased in human neural progenitor cells exposed to model air pollutants, indicating that both genetic and environmental factors that contribute to ASD increased RPS10P2-AS1 expression. Overexpression of RPS10P2-AS1 in human neural progenitor cells indicated substantial changes in neuronal gene expression. These data indicate that genome-wide significant associations with ASD implicate long noncoding RNAs. Because long noncoding RNAs are more abundant in human brain than protein-coding RNAs, this class of molecules is likely to contribute to ASD risk.

Keywords: epigenetics, brain, development, autism, IncRNA, noncoding RNA, genetics, environment 


\section{INTRODUCTION}

Autism spectrum disorder (ASD) is a neurodevelopmental disorder that is characterized by deficits in social communication and behavioral flexibility (Levitt and Campbell, 2009). ASD symptoms begin by age 3, last throughout the lifespan, and impact $1.5 \%$ of the population (Beversdorf and MISSOURI AUTISM SUMMIT CONSORTIUM, 2016). The causes of ASD are an active area of research. Although de novo loss of function mutations have been highlighted in the genetics of ASD, it is clear that the majority of ASD genetic influences are from common genetic variants of relatively small effect (Anney et al., 2012; Gaugler et al., 2014). Association of common genetic variants is most efficiently identified by genome-wide association studies (GWAS). The first published GWAS for ASD reported genomewide significant association $\left(\mathrm{P}<5 \times 10^{-8}\right)$ of genetic markers on chromosome 5p14.1 (Wang et al., 2009). These markers mapped between two protein-coding genes known to be involved in brain development. However, we recently reported that the functional element implicated by the chromosome 5p14.1 genetic markers was a long noncoding RNA, Moesin Pseudogene 1 Anti-Sense (MSNP1AS) (Kerin et al., 2012). The expression of MSNP1AS was increased in postmortem cerebral cortex of both individuals with ASD and individuals with the genotypes associated with ASD; the expression of neither of the neighboring chromosome 5 p14.1 protein-coding genes was altered in ASD or correlated with the genotypes associated with ASD (Kerin et al., 2012). We therefore concluded that MSNP1AS was the functional element revealed by the chromosome 5p14.1 ASD genome-wide association peak (Kerin et al., 2012). We later found that overexpression of MSNP1AS in human neural progenitor cells altered neuronal morphology and expression of genes involved in two biological processes: protein synthesis and chromatin remodeling (DeWitt et al., 2016a). Similarly, transcriptional gene silencing of MSNP1AS in human neural progenitor cells altered expression of genes involved in chromatin remodeling and immune response (DeWitt et al., 2016b). The biological processes implicated by altered MSNP1AS expression in human neural progenitor cells are reminiscent of those implicated by rare de novo loss-offunction mutations, and thus suggest a convergent biology of ASD implicated by divergent genetic association approaches (DeWitt et al., 2016a; DeWitt et al., 2016b).

The second published GWAS for ASD reported genome-wide significant association of a single genetic marker, rs4141463, on chromosome 20p12.1 (Anney et al., 2010). The rs4141463 marker lies within an intron of the protein-coding gene MACRO domain containing 2 (MACROD2). Based on our previous findings of implication of the long noncoding RNA MSNP1AS by the chromosome 5 p14.1 genome-wide association instead of the neighboring protein-coding genes, we hypothesized that the chromosome 20p12.1 genome-wide association might also implicate a genetic element other than protein-coding MACROD2 gene. Bioinformatics approaches indicated the presence of a long noncoding RNA, Ribosomal Protein S10 Pseudogene 2 AntiSense 1 (RPS10P2-AS1) at the site of the rs4141463 association with ASD. In this report, we provide evidence that RPS10P2-AS1 is the functional element revealed by the chromosome 20p12.1
ASD genome-wide significant association signal and begin to determine the functions of RPS10P2-AS1 in human neural progenitor cells.

\section{MATERIALS AND METHODS}

Postmortem brain samples: Postmortem brain samples from 10 individuals with ASD and 10 age- and gender-matched controls were obtained from the Autism Tissue Program, now known as Autism BrainNet. All samples were Brodmann Area 22, corresponding to the superior temporal gyrus. Specific information about age- and gender-matching are listed in Table S1. Genotypes at the rs4141463 locus for each of the brain samples was determined by PCR amplification and Sanger sequencing.

Cell Culture: The human neuronal progenitor cell ReNcell CX was used in this study as the cells differentiate into cortical projection neurons (EMD Millipore, Darmstadt, Germany). ReNcell CX cells were maintained in tissue culture flasks at $37^{\circ} \mathrm{C}$ and $5 \% \mathrm{CO} 2$ in ReNcell NSC Maintenance Medium supplemented with FGF and EGF in laminin-coated flasks. Diesel particulate matter was purchased from Sigma, serially diluted in a small volume of dimethyl sulfoxide (Sigma), and added to the ReNcell medium for $24 \mathrm{~h}$ prior to harvest.

Over-expression transfection: To determine the impact of RPS10P2-AS1 over expression on gene expression in human neuronal cells, RPS10P2-AS1 was PCR-amplified using the KOD Xtreme Hot Start kit (Novagen, Madison, WI, USA). PCR product was first cloned into pSTBlue-1 then sub-cloned into pIRES2-AcGFP1 and pIRES2-DsRed2 vectors (Clonetech, Mountain View, CA, USA) using restriction sites to create sense and antisense clones. PCR product and vectors were sequence verified at multiple points during the cloning process. Cells were then grown to $\sim 70 \%$ confluency and cultured $24 \mathrm{~h}$ prior to experimentation. Cells were transfected with RPS10P2-AS1 and the reverse complement control (RPS1OP2) over-expression vectors using Amaxa Nucleofector kit (Lonza, Basel, Switzerland). Empty pIRES2 vectors were used as controls. We optimized the transfection experiments by manipulating the mass of plasmid transfected $(1 \mathrm{mg})$ and the duration of transfection (Amaxa program T-16) to achieve a consistent 8-fold increase in RPS10P2-AS1 in ReNcell CX cells after $24 \mathrm{~h}$. Experiments were harvested at $24 \mathrm{~h}$ post-transfection and were performed in quadruplicate. Prior to RNA sequencing, qPCR was used to confirm over-expression of RPS10P2-AS1 by 7.7- to 8.3-fold.

RNA Isolation: Experimental cells were harvested and pelleted by centrifugation. Cell pellets were homogenized using Qiashredder spin columns (Qiagen, Valencia, CA, USA) and then total RNA was extracted using Qiagen RNeasy kit according to the manufacturer's protocol. Additionally, total RNAs (Biochain, Newark, CA, USA) were purchased to look at expression in tissues of other areas of the human body. Total RNAs purchased include: Adult Normal Tissue Brain Temporal Lobe (R1234078-50), Adult Normal Tissue Brain Cerebellum (R1234039-50), Adult Normal Tissue Brain Frontal Lobe (R1234051-50), Adult Normal Tissue Brain Occipital Lobe (R1234062-50), Adult Normal Tissue Spinal Cord (R1234234-50), Adult Normal Tissue Peripheral Blood Leukocyte (R1234148-10), 
Adult Normal Tissue Heart (R1234122-50), Adult Normal Tissue Lung (R1234152-50), Adult Normal Tissue Kidney (R1234142-50), Adult Normal Tissue Skin (R1234218-50), Fetal Normal Tissue Brain Temporal Lobe (R1244078-50), Fetal Normal Tissue Brain Frontal Lobe (R1244051-50), Fetal Normal Tissue Heart (R124412250), Fetal Normal Tissue Lung (R1244152-50) and Fetal Normal Tissue Kidney (R1244142-10).

Quantitative RT-PCR: RNA extracted samples were converted to cDNA using Life Technologies Superscript III RT-PCR kit (Life Technologies, Carlsbad, CA, USA). Then qPCR was performed on a Life Technologies StepOnePlus PCR System using the following TaqMan gene expression assays: RPS10 (Hs01693877_ s1), RPS10P2-AS1 (AI1RVBK), GAPDH (Hs9999905_m1) and POL2RA (Hs00172187_m1). GAPDH and POLR2A serve as housekeeping gene controls. Data was analyzed via StepOnePlus software v2.3.

RNA-seq: RNA-seq was performed in order to determine which genes would be differentially expressed as a result of RPS10P2-AS1 over-expression. First total RNA quality was assessed by using a Nanodrop ND-1000 Spectrophotometer (Thermo Fisher Scientific, Waltham, MA, USA) to look at 260/280 absorbance ratios then using an Agilent Technologies 2200 TapeStation Instrument (Agilent Technologies, Santa Clara, CA, USA) to determine RNA integrity numbers. Next cDNA libraries were constructed from RNA extracted from cell pellets with the Illumina Truseq Stranded Total RNA Sample Preparation kit and sequencing was performed on the HiSeq2000 sequencer to generate $101 \mathrm{bp}$ single-end reads (Illumina, San Diego, CA, USA). The average number of reads per sample run was 36 million reads. Each RPS10P2-AS1 transfection was performed in quadruplicate. RNA-seq was performed on each of the four transfection trials.

RNA-seq analysis: RNA sequencing analysis was performed as previously described (Wilkinson et al., 2015). Briefly, sample files were run though a quality control pipeline by first removing adapters from raw reads with the Cut Adapt program (Martin, 2011). Then sample files were aligned to available genome builds (UCSC Hg19, Ensembl GrCH37 and Ensembl GrCH38) using Tophat2 (Kim et al., 2013). After alignment, fastq files were run through the FastQC program to further assess quality of files 12. Once samples were mapped differential gene expression analysis was performed using Cuffdiff with corresponding transcriptomes under default settings which compared RPS10P2-AS1 overexpression vector to empty vector controls at each time point per cell line (Trapnell et al., 2012). A list of significantly differentially expressed genes was entered into the Database for Annotation, Visualization and Integrated Discovery (DAVID) for gene enrichment analysis for each cell line at each time point (Huang et al., 2009). It is important to note that Ensembl builds inherently contain more ncRNAs than the UCSC build, hence, all builds were examined in depth. We report here both $P$ values that are corrected for multiple comparisons ( $q$ values) and $\mathrm{P}$ values that are uncorrected for multiple comparisons ( $\mathrm{p}$ values). All discussions of individual genes are those that are $\mathrm{q}$ value significant (i.e., significant after correction for multiple comparisons). However, all discussions of downstream gene ontology (DAVID) analyses include all genes that are significant without correction for multiple comparisons.

\section{RESULTS}

The single nucleotide polymorphism (SNP) rs4141463 was assigned genome wide association with ASD through a GWAS 9. The rs4141463 SNP is within an intron of the MACROD2 gene. However, MACROD2 spans more than 2 million base pairs (2 Mbp) of chromosome 20, and rs4141463 resides within a particularly large intron that spans more than 500,000 base pairs $(500 \mathrm{~kb})$ of DNA sequence. Within the intron of MACROD2 is the 494 bp long noncoding RNA RPS10P2-AS1, which resides just $9 \mathrm{~kb}$ from rs4141463. Due to the proximity of both MACROD2 and RPS10P2-AS1 to the ASD genome wide significant signal, we decided to test both genes as quantitative trait loci for rs4141463.

Publicly available gene expression data from the GTex consortium indicated that both MACROD2 and RPS10P2-AS1 are expressed at detectable levels in human cerebral cortex (GTEx Consortium, 2015). To get a more detailed map of expression for both genes, we performed qPCR for both MACROD2 and RPS10P2-AS1 in a panel of tissues that included several regions of cerebral cortex (Figure 1). Our qPCR data indicated that both transcripts are expressed at detectable levels in each of the 10 adult and 5 fetal tissues (Figure 1). RPS10P2-AS1 expression was highest in fetal temporal cortex, fetal frontal cortex and adult cerebellum (Figure 1). MACROD2 expression levels were highest in fetal frontal cortex, fetal temporal cortex, and adult lung (Figure 1). Expression of RPS10P2-AS1 was higher than MACROD2 in 9 of the 15 tissues, including 4 of the 5 fetal tissues (Figure 1).

We next tested the expression of MACROD2 and RPS10P2-AS1 in postmortem temporal cerebral cortex, a region of the brain in which both transcripts are expressed in both fetal and adult tissue (Figure 1), of 10 individuals with ASD and 10 non-ASD controls. The expression of MACROD2 was not significantly different between individuals with ASD and controls (Figure 2). In contrast, there was a 7-fold increase in average RPS10P2-AS1 expression in postmortem temporal cortex of individuals with

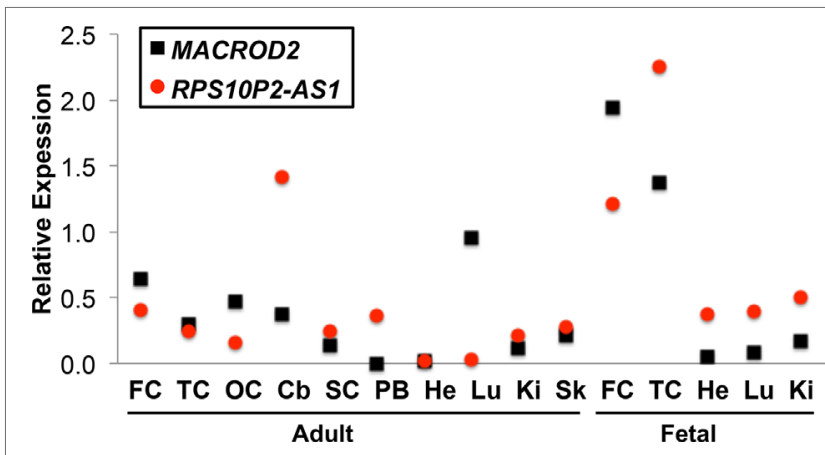

FIGURE 1 | Relative expression of MACROD2 and RPS1OP2-AS1 in 15 human tissues. Although noncoding RNAs are often expressed at lower levels than protein-coding genes in bulk tissue, RPS10P2-AS1 is expressed at higher levels than MACROD2 in all 15 tissue samples tested. RPS1OP2-AS1 is expressed at higher levels than MACROD2 in adult cerebellum, spinal cord, peripheral blood, kidney and skin and in fetal temporal cortex, heart, lung, and kidney. Each qPCR was performed 4 times and the results averaged. FC, frontal cortex; TC, temporal cortex; OC, occipital cortex; $\mathrm{Cb}$, cerebellum; SC, spinal cord; $\mathrm{PB}$, peripheral blood; $\mathrm{He}$, heart; Lu, lung; Ki, kidney; Sk, skin. 

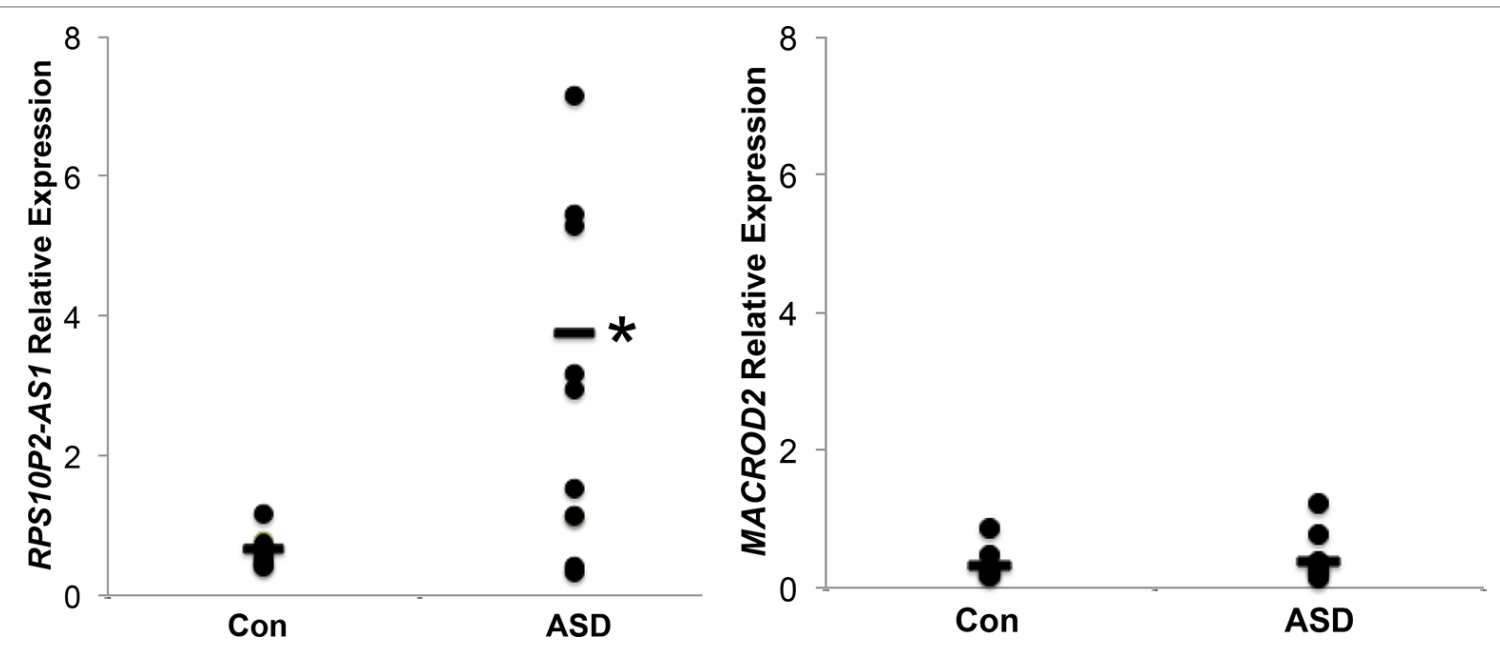

FIGURE 2 | Relative expression of RPS10P2-AS1 and MACROD2 in postmortem temporal cortex of 10 individuals with ASD and 10 age- and gender-matched controls. While there is no significant change in MACROD2 expression in postmortem brain of individuals with ASD, there is a 7-fold increase in RPS10P2-AS1 in individuals with ASD compared to controls $(P<0.01)$. Each qPCR was performed 4 times and the results averaged. As should be expected from a disorder with multiple etiologies, half of the ASD brain samples show levels of RPS10P2-AS1 that is comparable to the controls; however, half of the ASD brain samples show RPS10P2-AS1 expression substantially higher than any of the controls. * indicates $\mathrm{P}<0.01$ by Student's t-test.

ASD compared to age- and gender-matched controls (Figure 2; $\mathrm{P}=0.01$ ). These data indicate that RPS10P2-AS1 expression, but not MACROD2 expression, is associated with ASD diagnosis.

To determine the association between rs4141463 genotype and expression of MACROD2 and RPS10P2-AS1 in human temporal cortex, we genotyped rs4141463 in the 20 human temporal cortex samples. The rs4141463 C allele is associated with ASD (Anney et al., 2010). In the postmortem temporal cortex samples, the rs4141463 C/C genotype was associated with a significantly higher expression of RPS10P2-AS1 than the T/T genotype (Figure 3). Individuals with the rs4141463 heterozygous genotype $\mathrm{C} / \mathrm{T}$ had an intermediate expression of RPS10P2-AS1 (Figure 3). In contrast to the significant association of rs4141463 genotype with RPS10P2-AS1 expression, there was no correlation between genotype and MACROD2 expression (Figure 3). These data indicate that RPS10P2-AS1 is the functional element revealed by
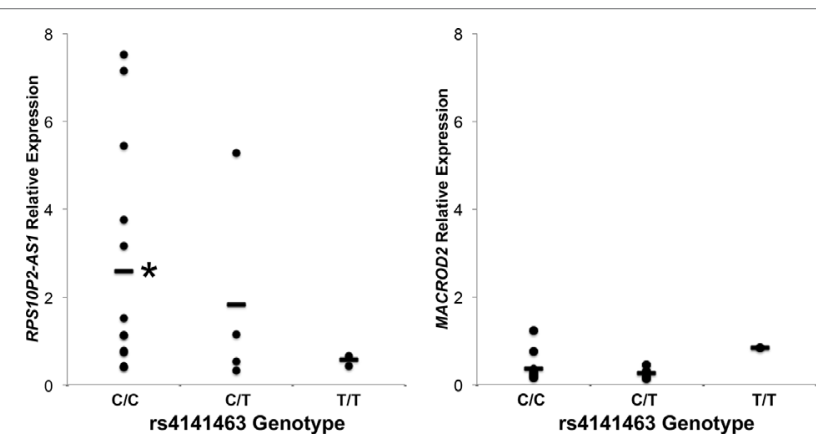

FIGURE 3 | Relative expression of RPS10P2-AS1 and MACROD2 by genotype at the rs4141463 locus in postmortem temporal cortex. The common rs4141463 C allele is associated with ASD by GWAS. The C allele is associated with higher expression of RPS10P2-AS1, but is not correlated with expression levels of MACROD2. $\mathrm{N}=4$. * indicates $\mathrm{P}<0.01$ by ANOVA followed by Tukey-Kramer test. genome wide significant association of rs4141463. GTEx analysis indicates that rs4141463 does not have a significant eQTL with any gene in any tissue. However, it should be noted that superior temporal cerebral cortex is not among the tissues tested in GTEx.

In addition to genetic factors, environmental factors can increase ASD risk. For example, near roadway air pollution can double the risk of ASD (Volk et al., 2013). To test the impact of a model air pollutant on RPS10P2-AS1, we exposed ReNcell CX human cortical projection neurons to increasing concentrations of diesel particulate matter (DPM). The DPM exposure caused a dose-dependent increase in RPS10P2-AS1 expression: $10 \mathrm{ng} / \mathrm{ml}$ DPM increased RPS10P2-AS1 by a non-significant 1.9 -fold; $20 \mathrm{ng} / \mathrm{ml}$ DPM increased RPS10P2-AS1 by a significant 4 -fold; and both 50 and $100 \mathrm{ng} / \mathrm{ml}$ DPM caused a significant 7-fold increase in RPS10P2-AS1 (Figure 4). In contrast, exposure of ReNcell CX cells to DPM did not significantly change the expression of the protein-coding gene MACROD2 (Figure 4). These data indicate that exposure of human cortical projection neurons to an ASD-associated environmental factor causes an increase in the noncoding RNA RPS10P2-AS1 that is on the same scale as the increased expression observed in postmortem cerebral cortex of individuals with ASD. The environmental response of $R P S 10 P 2-A S 1$ provides further suggestive evidence, in addition to the genetic evidence, that the lncRNA may contribute to ASD risk.

The expression of RPS10P2-AS1 was increased in postmortem brains of individuals with ASD (Figure 2), in postmortem brains of individuals with the ASD-associated rs4141463 C/C genotype (Figure 3), and in cultured human projection neurons exposed to DPM (Figure 4). To determine the impact of RPS10P2-AS1 overexpression on gene expression in developing human neurons, we transfected a RPS10P2-AS1 over-expression construct into ReNcell CX human cortical projection neurons and performed RNA sequencing to determine the impact on gene expression. We calibrated the protocol to over-expression at $\sim 8$-fold increased expression of RPS10P2-AS1, similar to the levels observed in 


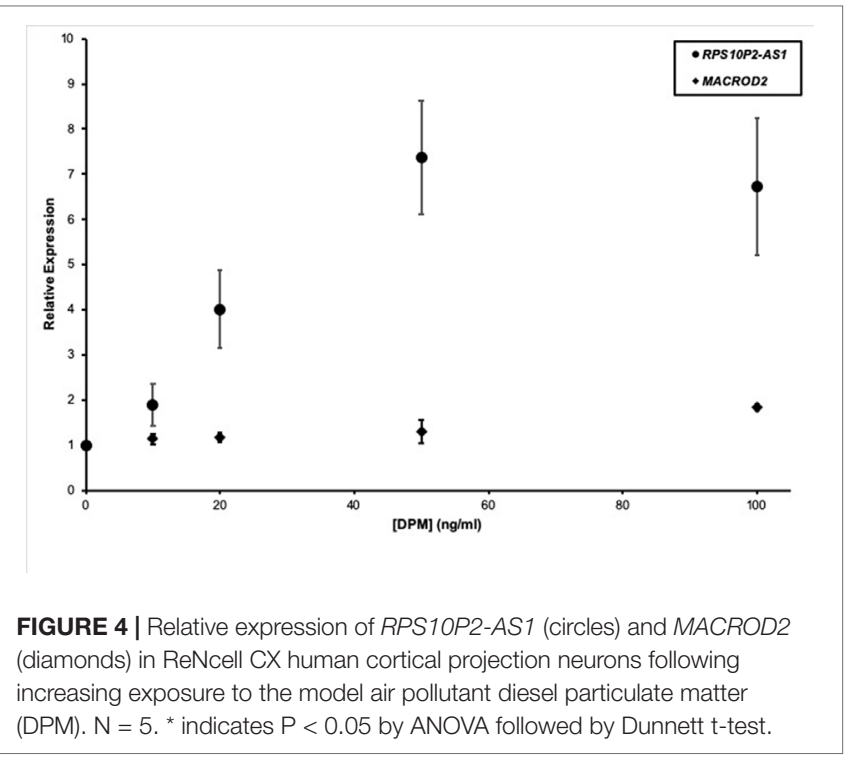

postmortem cerebral cortex of individuals with ASD and in ReNcell CX cells exposed to DPM. A total of 143 genes were differentially expressed following RPS10P2-AS1 over-expression ( $\mathrm{p}<0.05$, Supplementary Table 2 ), with 14 genes differentially expressed following correction for multiple comparisons $(\mathrm{q}<0.05$, Supplementary Table 2). The RNA-seq results were validated by qPCR of selected genes (Figure 5) and show a significant correlation between RNA-seq and qPCR (Pearson $\mathrm{R}=0.84 ; \mathrm{P}<1 \times 10^{-5}$ ). The most significant changes in gene expression involved interferon inducible proteins 6 and 44 (IFI6 and IFI44) and small nuclear RNAs 4-1 and 4-1 (SNU4-1 and SNU4-2) (Supplementary Table 2). Gene ontology analysis of the 143 differentially expressed genes $(\mathrm{P}<$ 0.05 ) indicated enrichment of genes involved in apoptosis, response to organic substances, neurotransmitter transport and synapses
(Figure 6). These data suggest that RPS10P2-AS1 may play a critical role in neuron development and response to environmental insults.

\section{DISCUSSION}

Our data indicate that the long noncoding RNA RPS10P2-AS1, not the protein-coding RNA MACROD2, is a functional element revealed by the genome wide significant association of rs4141463 with ASD risk. RPS10P2-AS1 expression is increased in postmortem temporal cortex of individuals with ASD. Increased RPS10P2-AS1 expression is correlated with the ASD-associated rs4141463 C allele. RPS10P2-AS1 expression is increased in human neural progenitor cells following exposure to ASD-associated diesel particulate matter. These data suggest that increased RPS10P2-AS1 may be a convergent molecular mechanism by which both common genetic variants and environmental factors contribute to increased ASD risk.

Genome wide association studies of ASD have identified several loci with genome wide significant association (Wang et al., 2009; Anney et al., 2010; Autism Spectrum Disorders Working Group of The Psychiatric Genomics Consortium, 2017; Grove et al., 2019). The chromosome 20p12.1 region is one of the few regions that has been implicated multiple times as a recent study identified genome wide significant association of rs71190156, which is $89 \mathrm{~kb}$ from rs4141463 and $98 \mathrm{~kb}$ from RPS10P2-AS1 (Autism Spectrum Disorders Working Group of The Psychiatric Genomics Consortium, 2017). Future experiments will be required to determine if association of rs71190156 implicates RPS10P2-AS1 or a different functional element on chromosome 20p12.1.

The identification of RPS10P2-AS1 as a functional element revealed by genome wide association of rs4141463 with ASD provides further evidence that long noncoding RNAs contribute to altered brain development. The default assumption has been that association of rs4141463 with ASD implicated MACROD2, the protein coding gene within which rs4141463 is located.

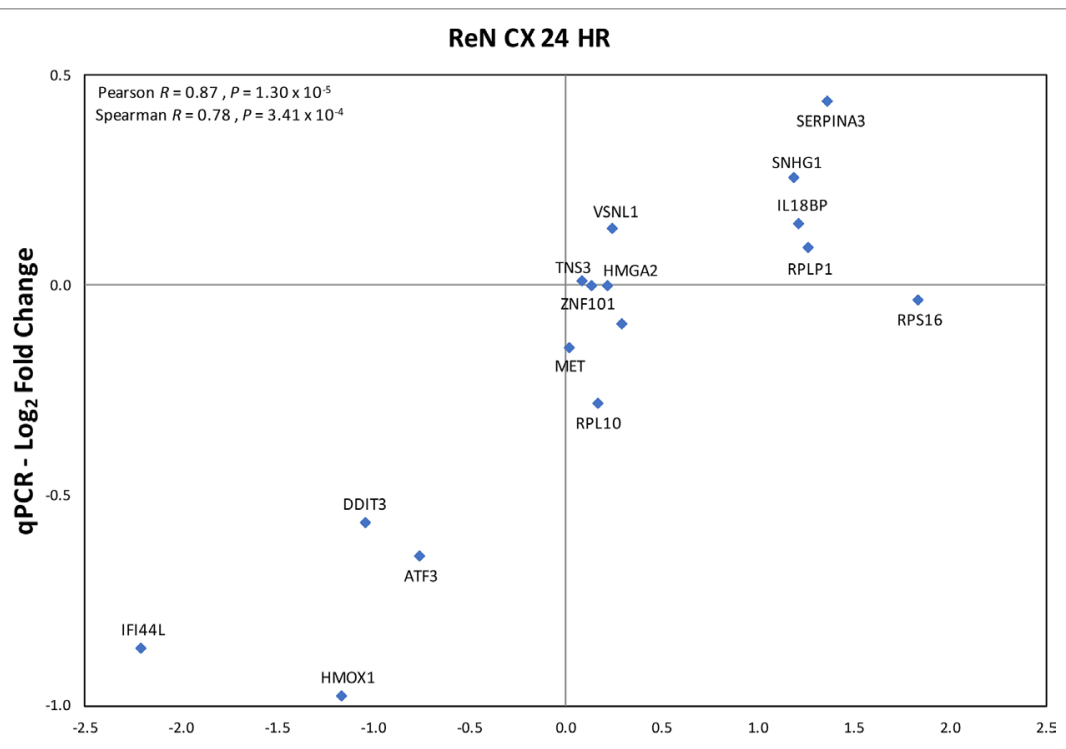

FIGURE 5 | Validation of RNA seq results by qPCR of selected genes in the same samples exposed to increased expression of RPS1OP2-AS1. N = 4. The qPCR results validate that RNA seq results with Pearson correlation $R=0.87\left(P=1.30 \times 10^{-5}\right)$. 


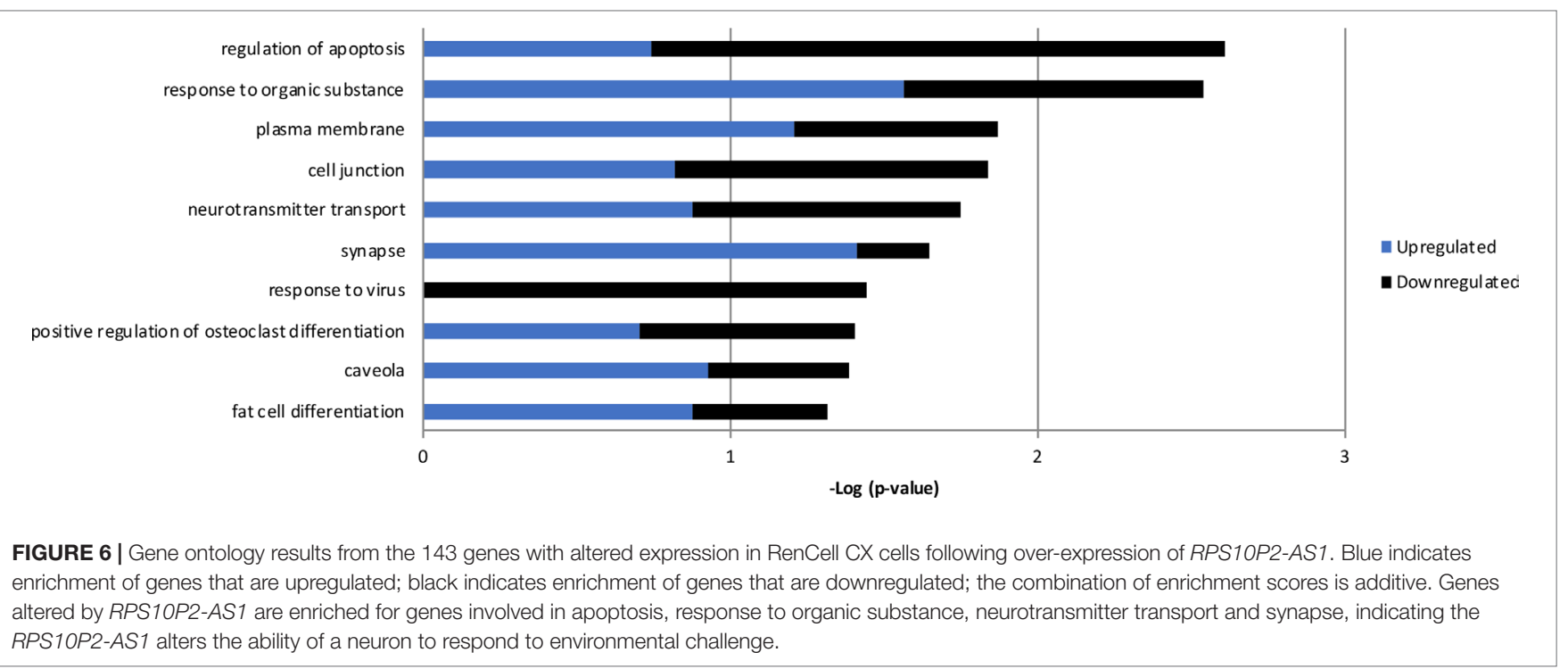

However, long noncoding RNAs account for half of the RNAs expressed in human brain (Kapranov et al., 2010) and human neural progenitor cells (Hecht et al., 2015). Therefore, it is not surprising that long noncoding RNAs are implicated in multiple neurodevelopmental disorders. We previously identified the long noncoding RNA MSNP1AS as a functional element revealed by genome wide significant association with ASD of chromosome 5p14.1 genetic markers (Kerin et al., 2012). The identification of RPS10P2-AS1 as the functional element revealed by rs4141463 association with ASD provides additional evidence of long noncoding RNA contribution to brain development.

Overexpression of RPS10P2-AS1 caused significant changes in the expression of 14 genes $(\mathrm{q}<0.05)$ in ReNcell CX cells. The zinc finger transcription factor ZNF865 was increased in expression more than 16-fold following RPS10P2-AS1 over-expression. The function of ZNF865 is unknown, as a PubMed search for ZNF865 yields no results. Overexpression of RPS10P2-AS1 increased the expression of a mitochondrial enzyme (RDH13), two small nuclear RNAs (RNU4-1 and RNU4-2), and two proteins involved in ribosomal function (RPS16 and RPLP1). Genes that decreased in expression following RPS10P2-AS1 overexpression include interferon-inducible genes (IFI6, MX1 and IFI44L), which may play a role in apoptosis, and genes involved in synthesis and shuttling of amino acids (SLC7A11 and ASNS). Gene ontology analysis of the 143 genes with $\mathrm{P}<0.05$ indicated significant enrichment of genes involved in the regulation of apoptosis, response to organic substances, neurotransmitter transport and synapses. This suggests that RPS10P2-AS1 may be involved in responses to environmental challenges that impact neuron function.

\section{DATA AVAILABILITY STATEMENT}

The raw data supporting the conclusions of this study can be found in NCBI using accession number PRJNA564157.

\section{AUTHOR CONTRIBUTIONS}

The experiments were conceived and designed by DC and NG. $\mathrm{SB}, \mathrm{KL}$, and NG performed the experiments and data analysis. DC wrote the first draft of the manuscript. All authors contributed to manuscript revision, and read and approved the submitted version.

\section{FUNDING}

This work was funded by the National Institute of Mental Health (R21MH099504), the National Institute of Environmental Health Sciences (R56 ES 029064), the Michigan State Foundation Discretionary Funds Initiative, and the Spectrum Health-MSU Alliance Corporation.

\section{ACKNOWLEDGMENTS}

Postmortem human cerebral cortex tissue was obtained from Autism BrainNet, which is sponsored by the Simons Foundation Autism Research Initiative. The authors also acknowledge the Autism Tissue Program, which was the predecessor to Autism BrainNet. The authors thank all brain donors for their valuable contribution to science.

\section{SUPPLEMENTARY MATERIAL}

The Supplementary Material for this article can be found online at: https://www.frontiersin.org/articles/10.3389/fgene.2019.00970/ full\#supplementary-material

SUPPLEMENTARY TABLE 1 | Description of donated postmortem brain samples obtained from the Autism Tissue Program, now Autism BrainNet. Each sample was from the superior temporal gyrus, which corresponds to Brodmann area 22. PMI, Post-Mortem Interval.

SUPPLEMENTARY TABLE 2 | List of 143 genes with significant changes ( $P<$ 0.05) in expression following over-expression of RPS1OP2-AS1 in ReNcell CX cells. 


\section{REFERENCES}

Anney, R., Klei, L., Pinto, D., Almeida, J., Bacchelli, E., Baird, G., et al. (2012). Individual common variants exert weak effects on the risk for autism spectrum disorders. Hum. Mol. Genet. 21, 4781-4792. doi: 10.1093/hmg/dds301

Anney, R., Klei, L., Pinto, D., Regan, R., Conroy, J., Magalhaes, T. R., et al. (2010). A genome-wide scan for common alleles affecting risk for autism. Hum. Mol. Genet. 19, 4072-4082. doi: 10.1093/hmg/ddq307

Autism Spectrum Disorders Working Group of The Psychiatric Genomics Consortium. (2017) Meta-analysis of GWAS of over 16,000 individuals with autism spectrum disorder highlights a novel locus at 10q24.32 and a significant overlap with schizophrenia. Mol. Autism 8, 21. doi: 10.1186/s13229-017-0137-9

Beversdorf, D. Q., and MISSOURI AUTISM SUMMIT CONSORTIUM. (2016). Phenotyping, Etiological Factors, and Biomarkers: toward precision medicine in autism spectrum disorders. J. Dev. Behav. Pediatr. 37, 659-673. doi: 10.1097/ DBP.0000000000000351

DeWitt, J. J., Grepo, N., Wilkinson, B., Evgrafov, O. V., Knowles, J. A., and Campbell, D. B. (2016a). Impact of the Autism-Associated Long Noncoding RNA MSNP1AS on Neuronal Architecture and Gene Expression in Human Neural Progenitor Cells. Genes (Basel) 7, 1-11. doi: 10.3390/genes7100076

DeWitt, J. J., Hecht, P. M., Grepo, N., Wilkinson, B., Evgrafov, O. V., Morris, K. V., et al. (2016b). Transcriptional Gene Silencing of the Autism-Associated Long Noncoding RNA MSNP1AS in Human Neural Progenitor Cells. Dev. Neurosci. 38, 375-383. doi: 10.1159/000453258

Gaugler, T., Klei, L., Sanders, S. J., Bodea, C. A., Goldberg, A. P., Lee, A. B., et al. (2014). Most genetic risk for autism resides with common variation. Nat. Genet. 46, 881-885. doi: 10.1038/ng.3039

Grove, J., Ripke, S., Als, T. D., Mattheisen, M., Walters, R. K., Won, H., et al. (2019). Identification of common genetic risk variants for autism spectrum disorder. Nat. Genet. 51, 431-444. doi: 10.1038/s41588-019-0344-8

GTEx Consortium. (2015). Human genomics. The Genotype-Tissue Expression (GTEx) pilot analysis: multitissue gene regulation in humans. Science 348, 648-660. doi: 10.1126/science.1262110

Hecht, P. M., Ballesteros-Yanez, I., Grepo, N., Knowles, J. A., and Campbell, D. B. (2015). Noncoding RNA in the transcriptional landscape of human neural progenitor cell differentiation. Front. Neurosci. 9, 392. doi: 10.3389/ fnins.2015.00392

Huang, D. W., Sherman, B. T., and Lempicki, R. A. (2009). Systematic and integrative analysis of large gene lists using DAVID bioinformatics resources. Nat. Protoc. 4, 44-57. doi: 10.1038/nprot.2008.211
Kapranov, P., St Laurent, G., Raz, T., Ozsolak, F., Reynolds, C. P., Sorensen, P. H. B., et al. (2010). The majority of total nuclear-encoded non-ribosomal RNA in a human cell is "dark matter" un-annotated RNA. BMC Biol. 8, 149. doi: 10.1186/1741-7007-8-149

Kerin, T., Ramanathan, A., Rivas, K., Grepo, N., Coetzee, G. A., and Campbell, D. B. (2012). A noncoding RNA antisense to moesin at 5p14.1 in autism. Sci. Transl. Med. 4, 128ra40. doi: 10.1126/scitranslmed.3003479

Kim, D., Pertea, G., Trapnell, C., Pimentel, H., Kelley, R., and Salzberg, S. L. (2013). TopHat2: accurate alignment of transcriptomes in the presence of insertions, deletions and gene fusions. Genome Biol. 14, R36. doi: 10.1186/gb-2013-14-4-r36

Levitt, P., and Campbell, D. B. (2009). The genetic and neurobiologic compass points toward common signaling dysfunctions in autism spectrum disorders. J. Clin. Invest. 119, 747-754. doi: 10.1172/JCI37934

Martin, M. (2011). Cutadapt removes adapter sequences from high-throughput sequencing reads. EMBnet.J. 17, 10-12. doi: 10.14806/ej.17.1.200

Trapnell, C., Roberts, A., Goff, L., Pertea, G., Kim, D., Kelley, D. R., et al. (2012). Differential gene and transcript expression analysis of RNA-seq experiments with TopHat and Cufflinks. Nat. Protoc. 7, 562-578. doi: 10.1038/nprot.2012.016

Volk, H. E., Lurmann, F., Penfold, B., Hertz-Picciotto, I., and McConnell, R. (2013). Traffic-related air pollution, particulate matter, and autism. JAMA Psychiatry 70, 71-77. doi: 10.1001/jamapsychiatry.2013.266

Wang, K., Zhang, H., Ma, D., Bucan, M., Glessner, J. T., Abrahams, B. S., et al. (2009). Common genetic variants on 5 p14.1 associate with autism spectrum disorders. Nature 459, 528-533. doi: 10.1038/nature07999

Wilkinson, B., Grepo, N., Thompson, B. L., Kim, J., Wang, K., Evgrafov, O. V., et al. (2015). The autism-associated gene chromodomain helicase DNA-binding protein 8 (CHD8) regulates noncoding RNAs and autism-related genes. Transl. Psychiatry 5, e568. doi: 10.1038/tp.2015.62

Conflict of Interest: The authors declare that the research was conducted in the absence of any commercial or financial relationships that could be construed as a potential conflict of interest.

Copyright (c) 2019 Bilinovich, Lewis, Grepo and Campbell. This is an open-access article distributed under the terms of the Creative Commons Attribution License (CC BY). The use, distribution or reproduction in other forums is permitted, provided the original author(s) and the copyright owner(s) are credited and that the original publication in this journal is cited, in accordance with accepted academic practice. No use, distribution or reproduction is permitted which does not comply with these terms. 